\title{
HADAMARD MATRICES OF ORDER 764 EXIST
}

\author{
DRAGOMIR Ž. ĐOKOVIĆ
}

Abstract. Two Hadamard matrices of order 764 of GoethalsSeidel type are constructed.

\section{Mathematics Subject Classification 05B20, 05B30}

Recall that a Hadamard matrix of order $m$ is a $\{ \pm 1\}$-matrix $A$ of size $m \times m$ such that $A A^{T}=m I_{m}$, where $T$ denotes the transpose and $I_{m}$ the identity matrix. We refer the reader to one of [2, 4] for the survey of known results about Hadamard matrices.

In our previous note [1], written about 13 years ago, we listed 17 integers $n \leq 500$ for which no Hadamard matrix of order $4 n$ was known at that time. Two of these integers were removed in that note and the smallest one, $n=107$, was removed recently by Kharaghani and Tayfeh-Rezaie [3]. Among the remaining 14 integers $n$ only four are less than 1000. The problem of existence of Hadamard matrices of these four orders, namely 668, 716, 764 and 892, has been singled out as Research Problem 7 in the recent book [2] by Kathy Horadam. In this note we shall remove the integer 764 from the mentioned list by constructing two examples of Hadamard matrices of Goethals-Seidel type of that order. (We have constructed a bunch of examples but we shall present only two of them.) Consequently, the revised list now consists of 13 integers:

167, 179, 223, 251, 283, 311, 347, 359, 419, 443, 479, 487, 491;

all of them primes congruent to $3(\bmod 4)$.

For the remainder of this note we set $n=191$. Let $G$ be the multiplicative group of non-zero residue classes modulo the prime $n=$ 191, a cyclic group of order $n-1=190$, and let $H=\langle 39\rangle=$ $\{1,39,184,109,49\}$ be its subgroup of order 5 . We choose the enumeration of the 38 cosets $\alpha_{i}, 0 \leq i \leq 37$, of $H$ in $G$ so that $\alpha_{2 i+1}=-1 \cdot \alpha_{2 i}$

The author was supported by the NSERC Grant A-5285. 
for $0 \leq i \leq 18$ and

$$
\begin{array}{lllll}
\alpha_{0}=H, & \alpha_{2}=2 H, & \alpha_{4}=3 H, & \alpha_{6}=4 H, & \alpha_{8}=6 H, \\
\alpha_{10}=8 H, & \alpha_{12}=9 H, & \alpha_{14}=11 H, & \alpha_{16}=12 H, & \alpha_{18}=13 H, \\
\alpha_{20}=16 H, & \alpha_{22}=17 H, & \alpha_{24}=18 H, & \alpha_{26}=19 H, & \alpha_{28}=22 H, \\
\alpha_{30}=32 H, & \alpha_{32}=36 H, & \alpha_{34}=38 H, & \alpha_{36}=41 H . &
\end{array}
$$

Define four index sets:

$$
\begin{aligned}
& J_{1}=\{1,7,9,10,11,13,17,18,25,26,30,31,33,34,35,36,37\}, \\
& J_{2}=\{1,4,7,9,11,12,13,14,19,21,22,23,24,25,26,29,36,37\} \\
& J_{3}=\{0,3,4,5,7,8,9,16,17,19,24,25,29,30,31,33,35,37\} \\
& J_{4}=\{1,3,4,5,8,11,14,18,19,20,21,23,24,25,28,29,30,32,34,35\}
\end{aligned}
$$

and introduce the following four sets of integers modulo 191:

$$
S_{k}=\bigcup_{i \in J_{k}} \alpha_{i}, \quad k=1,2,3,4 .
$$

Their cardinals $n_{k}=\left|S_{k}\right|=5\left|J_{k}\right|$ are:

$$
n_{1}=85, n_{2}=n_{3}=90, n_{4}=100
$$

and we set

$$
\lambda=n_{1}+n_{2}+n_{3}+n_{4}-n=174 .
$$

For $r \in\{1,2, \ldots, 190\}$ let $\lambda_{k}(r)$ denote the number of solutions of the congruence $i-j \equiv r(\bmod 191)$ with $\{i, j\} \subseteq S_{k}$. It is easy to verify (by using a computer) that

$$
\lambda_{1}(r)+\lambda_{2}(r)+\lambda_{3}(r)+\lambda_{4}(r)=\lambda
$$

is valid for all such $r$. Hence the sets $S_{1}, S_{2}, S_{3}, S_{4}$ are supplementary difference sets (SDS), with associated decomposition

$$
\begin{aligned}
4 n=764 & =9^{2}+11^{2}+11^{2}+21^{2} \\
& =\sum_{k=1}^{4}\left(n-2 n_{k}\right)^{2} .
\end{aligned}
$$

Let $A_{k}$ be the $n \times n$ circulant matrix with first row

$$
a_{k, 0}, a_{k, 1}, \ldots, a_{k, n-1}
$$

where $a_{k, j}=-1$ if $j \in S_{k}$ and $a_{k, j}=1$ otherwise. These $\{ \pm 1\}$-matrices satisfy the identity

$$
\sum_{k=1}^{4} A_{k} A_{k}^{T}=4 n I_{n} .
$$

One can now plug in the matrices $A_{k}$ into the Goethals-Seidel array to obtain a Hadamard matrix of order $4 n=764$. 
Our second example is constructed in the same way by using the index sets:

$$
\begin{aligned}
J_{1}= & \{0,1,6,8,9,11,12,16,18,20,21,23,28,31,33,36,37\}, \\
J_{2}= & \{0,1,3,4,10,12,13,17,20,22,24,31,32,33,34,35,36,37\}, \\
J_{3}= & 4,8,9,10,12,13,14,16,17,20,21,24,26,27,29,31,32,34\}, \\
J_{4}= & 1,7,9,10,11,12,14,15,16,17,20,22,23,25,28,29,32,33,34, \\
& 37\} .
\end{aligned}
$$

The two solutions are not equivalent in the sense that the two SDS's are not equivalent. (For the definition of equivalence for SDS's see our note [1].)

\section{REFERENCES}

[1] D.Ž. Đoković, Two Hadamard matrices of order 956 of Goethals-Seidel type, Combinatorica 14 (1994), 375-377.

[2] K.J. Horadam, Hadamard matrices and Their Applications, Princeton University Press, 2007.

[3] H. Kharaghani and B. Tayfeh-Rezaie, A Hadamard matrix of order 428, J. Combin. Designs 13 (2005), 435-440.

[4] J. Seberry and M. Yamada, Hadamard matrices, sequences and block designs, in "Contemporary Design Theory, A Collection of Surveys", J.H. Dinitz and D.R. Stinson, Eds., J. Wiley, New York, 1992.

Department of Pure Mathematics, University of Waterloo, WaterLOO, OntaRio, N2L 3G1, CANADA

E-mail address: djokovic@uwaterloo.ca 